\title{
S57. Computers in mental health
}

DEVELOPING A COMMON LANGUAGE FOR COMPUTER PROGRAMMES: ICD-10

H. J. Freyberger, C.P. Malchow, H. Dilling

Department of Psychiatry, Medical University Lübeck, Ratzeburger Allee 160, 23538 Lübeck, Germany

ICD-10, Chapter V(F) Mental and Behavioural Disorders is introducing an international standard of operationalized diagnoses on several purposes for users. The differenciation on different levels of operationalization in the Clinical and Diagnostic Guidelines, the Diagnostic Criteria for Research (DCR) and the Primary Health Care Classification (PHC) are the necessary basis for the development of different computing instruments in psychiatry. A masterplan, integrating the computerized applications of diagnostic instruments like the "Composite International Diagnostic Interview (CIDI)" and the "Schedules for Assessments in Neuropsychiatry (SCAN)" has the objective to provide additionally as a basic element for all computing instruments computerized ICD-10 textbooks. Therefore the development of a computer tutorial using the hypertext approach and WINDOWS help facility will be discussed with regard to general requirements of software and hardware elements.

\section{DEVELOPING A CLINICAL EXAMINATION TOOL} THE SCAN COMPUTER PROGRAM

S. Chatterii, R.Y. Mehta, A.Y. Tien, B. Üstün

Multiple approaches have been used in making diagnoses in psychiatry. We have come a long way from impressionistic approaches to operationalised systems in order to reduce individual idiosyncracies and facilitate standardization. The Schedules for Clinical Assessment on Neuropsychiatry (SCAN) is one such clinical interview that generates a symptom base and then builds up towards a diagnosis using the criteria specified in the International Classification of Diseases (10th ed), Diagnostic Criteria for Research (ICD-10 DCR) and the Diagnostic and Statistical Manual of the American Psychiatric Association 4th edition (DSM 4). The SCAN is an extremely useful tool for the clinician. However, to be able to use the instrument to its maximum potential and improve efficiency, the instrument needs to be computerized so that a large clinical data base can be generated, diagnoses easily checked and complex analysis easily performed. The computerization of the interview is described in this paper. The interview has been developed on a Windows platform where the interview text, the glossary and the rating boxes are available on screen. This greatly improves the efficiency of using SCAN, which has grown to become unwieldy in its printed form. This will in turn make the objectives of SCAN more easily obtainable, l.e. international standardization of assessment and diagnosis in psychiatry. Object oriented programming permits the linking of these easily. On line help and a tutorial are available. The ICD-10 DCR and DSM-4 criteria can be called up easily. Data can be represented graphically. The data is stored in a standard format and can be exported to a statistical analysis package. Scales like the Brief Psychiatric Rating Scales, the Hamilton Depression Rating Scale and the Scale for the Assessment of Negative Symptoms can be derived. This facilitates efficient, error free collection of data and saves times and permits large scale data management. 
A ROBUST EPIDEMIOLOGIC TOOL : CIDI-AUTO

CB Pull

Service de Psychiatrie, Centre Hospitalier de Luxembourg, 4 rue

Barblé, L-1210 Luxembourg, Luxembourg.

The Composite International Diagnostic Interview is a fully structured diagnostic interview which was developed as a collaborative project between the World Health Organization and the US Alcohol, Drug Abuse and Mental Health Administration. It is the most widely used structured diagnostic interview in the world. Version 1.0 was released in December 1990 and Version 1.1 in May 1993.

The Sydney Centre has produced a computerized CIDI, known as CIDI-Auto, for the World Health Organization. CIDI-Auto 1.0 was released in June 1992 and proved robust in use at 40 sites. The computerized version of CIDI-Auto 1.1 is now available. CIDI-Auto 1.1 can be self-administered or interviewer-administered. CIDI-Auto is being translated into Dutch, French, German, Portuguese and Spanish. The validity of CIDI-Auto 1.0 has been established and the reliability and validity of CIDi-Auto 1.1 is being assessed. The principal advantages of the computerized version are increased reliability of administration, and a significant reduction in the staff time required to administer the interview and enter and score the data.

The CIDI-Auto will be demonstrated and the use and validity of this method will be discussed.

\section{A STRATEGY FOR MENTAL HEALTH INFORMATICS \\ JK Wing \\ Royal College of Psychiatrists Research Unit \\ 11 Grosvenor Terrace, London 5W1X 7EE, UK}

Health service managers, clinicians and patients (probably in this order) are gradually coming to recognise the advantages to be gained from standardising and computerising core parts of the health record. The advantages are maximised if clinical work is carried out according to the principles of the needs assessment cycle: problems are assessed interventions chosen for each problem, trained staff allocated to provide each form of care, and appropriate settings for staff-patient interaction provided. A cost can be allocated to each item of care. Repeating the initial assessment after an interval allows outcomes to be measure. Guidelines based on state of the art knowledge are used at each point in the cycle and clinical audit is facilitated.

Technology is sufficiently advanced to support these functions and clinicians are thereby rewarded if they use such health information systems. The information collected is of good quality because it is needed for clinical use.

When anonymised and aggregated the same information will also serve public health functions (epidemiology, health services research, process and outcome measurement, and quality assurance) and administrative purposes (planning, contracts and resource management).

A coherent mental health informatics strategy is therefore based on simple principles. Information is person-based, generally entered on a computer only once, derived from systems used by clinicians rather than administrative staff, easily transferable in order to create continuity of care, and subject to high standards of security and confidentiality.

Clinicians should play a full part in the creation of such 'bottom-up' systems, firmly based on the needs of patients. These should replace the current 'top-down' management- driven systems. Work already in progress towards this end will be reviewed.

Developing Comprehensive Computerized Diagnosis-ClassificationManagement Systems

\section{INFORMATION TECHNOLOGY AS AN AID TO MENTAL HEALTH CARE Marks \\ Institute of Psychiatry, London SE58AF, UK}

Information technology (IT) systems can now improve mental health care and allow untreated sufferers access at affordable cost. Self-care is a key element in behaviour therapy of anxiety and other disorders, and can be instructed by a computer as well as by a clinician. Computer systems will be available by the end of 1994 to assist self-care of general anxiety, of phobic/panic disorders, and of obsessive-compulsive disorder. A computer system (CORM - Clinical Outcome and Resource measure) will also be avalable then to expedite the collection of quick and reliable performance specifications about treatment outcome and its cost. This can enhance the treatment process and outcome. Such benefit/cost audit can be incorporated into routine care and treatment research. IT aids are an attractive way to support clinical decisions, expedite mental health care, lower its unit cost, reduce the stigma of psychiatric referral, and give access to care to many sufferers who are currently denied it. according to a structured format. A check list of functions were
T.B. Üstün

World Health Organization, Division of Mental Health. Geneva, Switzerland

OBJECTIVES: This paper reviews the basic features of computerized information systems for the diagnosis, classification and management of the mental disorders. In parallel to the work of WHO's Division of Mental Health on ICD-10 and related family of instruments several computer applications have been developed for diagnosis, classification and management of mental disorders.

METHODS: The review is based on the operational computerized information systems that has been developed in collaboration with WHO. Developer of each system was asked to present their system specifically developed for the review and filled in by a panel of experts.

STUDY RESULTS: The results are reported within the perspective of articulating a comprehensive computerized diagnosis-classification and management system. There is a wide range of possibilities which cover a large number of programmes, and technological advances call for a plan that brings different parts fit each other harmoniously; similar to a "lego system": hence the need for standards for developing programmes and policy principles to govern future trends. These tools may assist in closing the gap between the developed and developing countries considering the cost-effective way of dissemination of information and communication.

CONCLUSION: Computer applications for mental health are developing rapidly. They could change provision of mental health care beyond recognition within a generation. Mental health expertise and information resources can be provided more widely on computers with improved quality. 